\title{
Design and implementation of Statistical Process Control based on MES Liu Sha ${ }^{1, \text { a }}$, Li Peng ${ }^{1}$, Hu Guolinag ${ }^{1}$, Duan Bin ${ }^{1}$
}

\author{
Research Laboratory of Digital Factory, Shenyang Institute of Automation, Chinese Academy of \\ Science, Shenyang, 110186, China
}

aemail: liusha@sia.cn

Keywords: SPC, Quality Management, MES

\begin{abstract}
According to the principle of SPC control chart, combined with the application module and service module of MES system, the SPC based MES system is realized, which contains Generation Control Chart sub module, Test Control Chart sub module, Product Field Monitor sub module, Data Acquisition sub module, out of Control Rules sub module.
\end{abstract}

\section{Introduction}

Digital workshop technology [1] is the key step to implement Digital manufacturing. In the fierce competition of global economy, the quality of the product requirement of manufacturing enterprise get higher than ever [2]. Information and data can not only reflect the quality of products, but also can reflect a lot of enterprise [3].

In the production process, due to the influence of human, machine, material, method, environment, measurement and other factors, the fluctuation of product quality characteristic value cannot be avoided. Volatility is divided into two types, normal fluctuations and abnormal fluctuations [4].

\section{Statistical Process Control and SPC Algorithm}

The random fluctuation is caused by some accidental factors in the process of production. These factors are inevitable. It is difficult to measure the elimination of technology, but has little effect on the quality of the product [5] [6].

Abnormal fluctuation, also known as the system fluctuation, is caused by some abnormal factors in the production process, which usually causes the product quality to change periodically or the tendency [7]. These factors are avoided, not with randomness, technology is easy to measure can be eliminated, and the quality of the product has a great impact, to meet the needs of consumers, must be eliminated [8].

\section{Statistical Process Control}

Statistical Process Control (SPC) is a technology of transforming data into information using data statistical analysis. SPC Chart is a graph designed with statistical methods, which is to determine, record, evaluate and monitor the quality of the process.

2. SPC Classification

Classification by data types, Statistical Control Chart can be divided into Variable Data SPC and Attributes Data SPC. Variable Data contains length, thickness, temperature, humidity, etc. Attributes data contains

Variable Data SPC contains Average-Range SPC ( $\bar{x}-R$ Chart), Average-Standard Deviation SPC ( $\bar{x}-s$ Chart), Median-Range SPC ( $x_{m}-R$ Chart), etc. Attributes Data SPC mainly contains Percent of Defective SPC ( $p$ Chart), Number of Defective SPC ( $n p$ Chart), etc. Here's the formula of $\bar{x}-R$ Chart as an example of SPC function.

\section{Statistical Process Control Implementation}

In this MES system, SPC contains Generation Control Chart, Test Control Chart, Product Field Monitor, Data Acquisition, Out of Control Rules sub module. The process of SPC interact with user 
as follow:

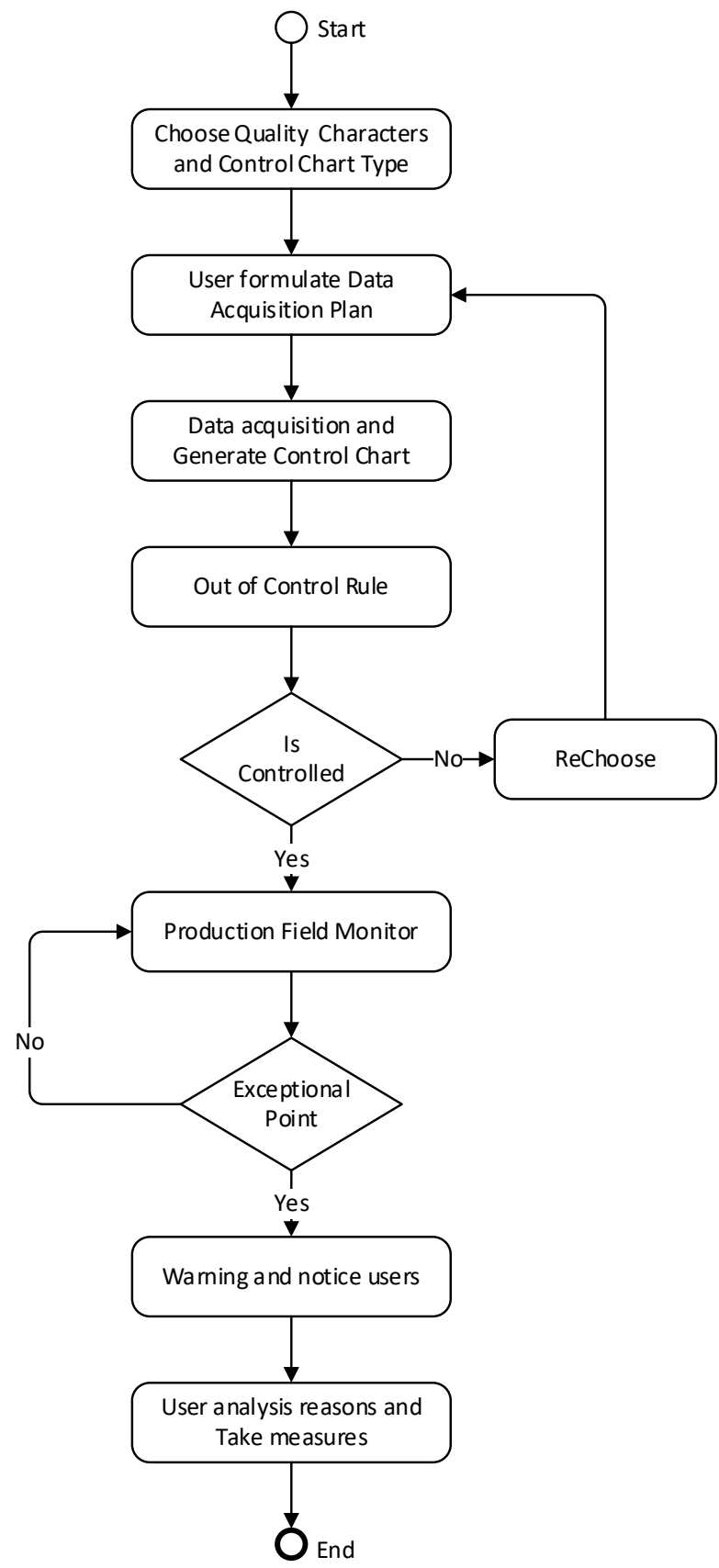

Fig. 1 SPC Interaction Process

User select control chart type, through the MES system configuration, to help users specify data acquisition program. Thereafter, the SPC Module collects data and generates control charts. In the case of uncontrolled data, the system prompts the user sample anomalies, and guide the user to develop a sampling plan. Monitoring the production site data, vomiting abnormal control points, the system alarm notification users, user-friendly analysis of causes and take appropriate measures.

1. Generation Control Chart Sub Module

Generation control chart sub module is responsible for collecting user specified samples, according to sample data, calculate control limits and other key parameters. 


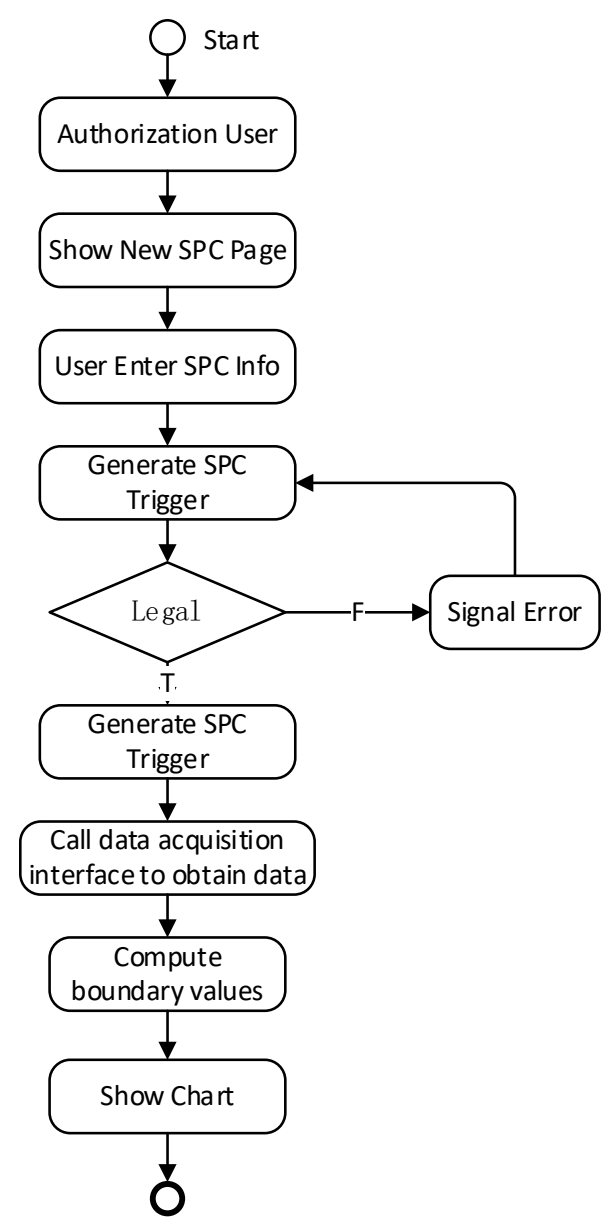

Fig. 2 Generation Control Chart Sub Module

- New control charts, the user needs to input or specify information include: control chart name, quality characteristics type, control chart type, sample data source, sample number, material variety, monitoring objectives, remarks, etc.;

- Legitimacy is mainly to determine whether the user input information conflict with each other (such as the choice of counting control charts, data sources are designated metering);

- The sample data source is the designated daily production plan, and the module then generates the sample data source according to the plan association to find the corresponding material quality inspection data;

- After sampling the samples, calculate the limits and key parameters according to the specified control chart type.

\section{Test Control Chart}

The new chart cannot be used directly for monitoring, and it needs to be verified. Only controlled control charts can be used to monitor on site production data. This SPC scheme provides a control chart test sub module for determining the controllability of control charts.

\section{Product Field Monitor}

When the control chart is verified, user monitors the quality of the material under production by field monitoring sub module. The process of product field monitor sub module as follow:

After the user specifies the control chart to trigger the production monitoring function, the system screens the batch according to the control chart material variety, displays the monitoring batch list. After the user selects the batch which needs to be monitored in the monitored batch list, the system reads the monitoring data of the changed batch material, depicts the data points in the control chart, and judges the discrimination rule. If there are abnormal points, alarm, and record a material. Does not appear abnormal point, the system automatically starts the timer, periodically 
monitor the batch status, until the batch completed.

4. Data Acquisition

The data acquisition sub module mainly provides the function of collecting data samples for the control chart.

\section{Out of Control Rules}

The discrimination rule sub module mainly combines the control chart to judge whether the sampling point is controlled.

Standard for judging exceptions: one of the following points is considered to be abnormal:

(1) The point is outside the control limit or just on the control limit;

(2) The arrangement of random points within the control limit is not random.

Inner point aligned random patterns include:

1) Multiple sample points appear on the centerline side continuously

- 11 consecutive points, there are at least 10 points in the side of the center line;

- 14 consecutive points, there are at least 12 points in the side of the center line;

- 17 consecutive points, there are at least 14 points in the side of the center line;

- 20 consecutive points, there are at least 16 points in the side of the center line;

Meet any one, the sample data is abnormal.

2) 7 consecutive points rise or fall

3) More boundary points

- 3 consecutive points in 2 - point fall in the warning area;

- 7 consecutive points in 3 - point fall in the warning area;

- 10 consecutive points in 4 - point fall in the warning area.

Meet an arbitrary, then the sample data is abnormal.

4) Periodic variation of sample points

5) Horizontal mutation of sample point distribution

6) The dispersion of sample points becomes large

\section{Statistical Process Control Application}

According to the MES system permission configuration framework structure, SPC function module and user interaction main role for quality administrator and technician. The main function module involves planning management module, scheduling module and WIP management module, including service for alarm service, database access service and resource coordination service.

The workflow of configurable SPC software based on MES system is divided into data sampling, generation control chart and monitoring production. The data sampling includes two kinds of online and offline sampling, online sampling refers to the sample data from the production site; off-line sampling refers to the sample data from the historical production record data.

- SPC query contains SPC rules entry and SPC query two page. Before the SPC query in this system, we should finish the entry of SPC rules.

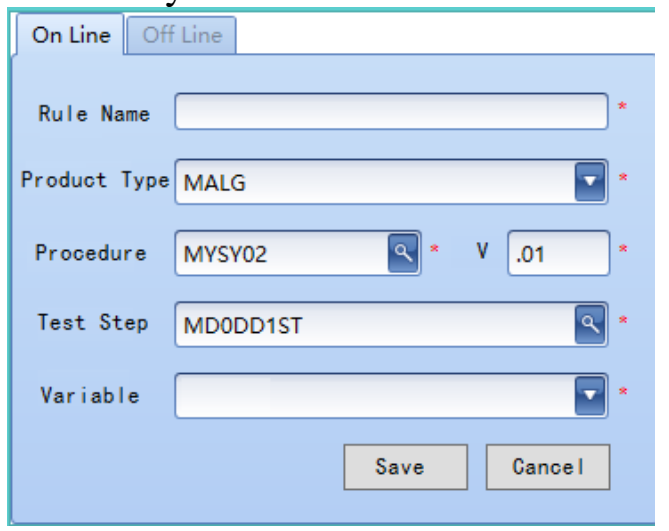

Fig. 3 SPC Rules 
SPC rules add windows, according to the needs of the entry needs of the SPC rules, including specifications, production varieties, process flow, test steps, variable groups and other information for the subsequent SPC query. Features include online SPC and offline SPC rules entry.

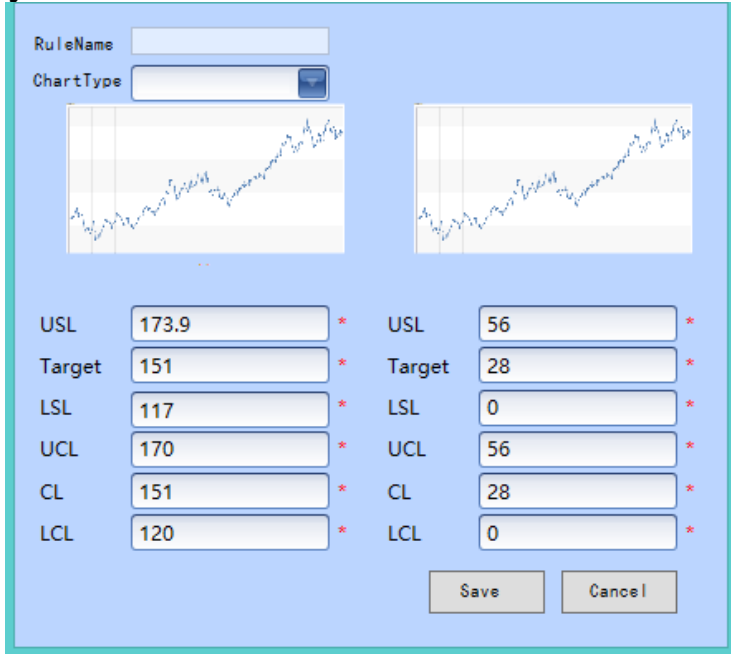

Fig. 4 Control Lines

- SPC query page, including SPC query, SPC control chart generation and change control chart display mode and other functions.

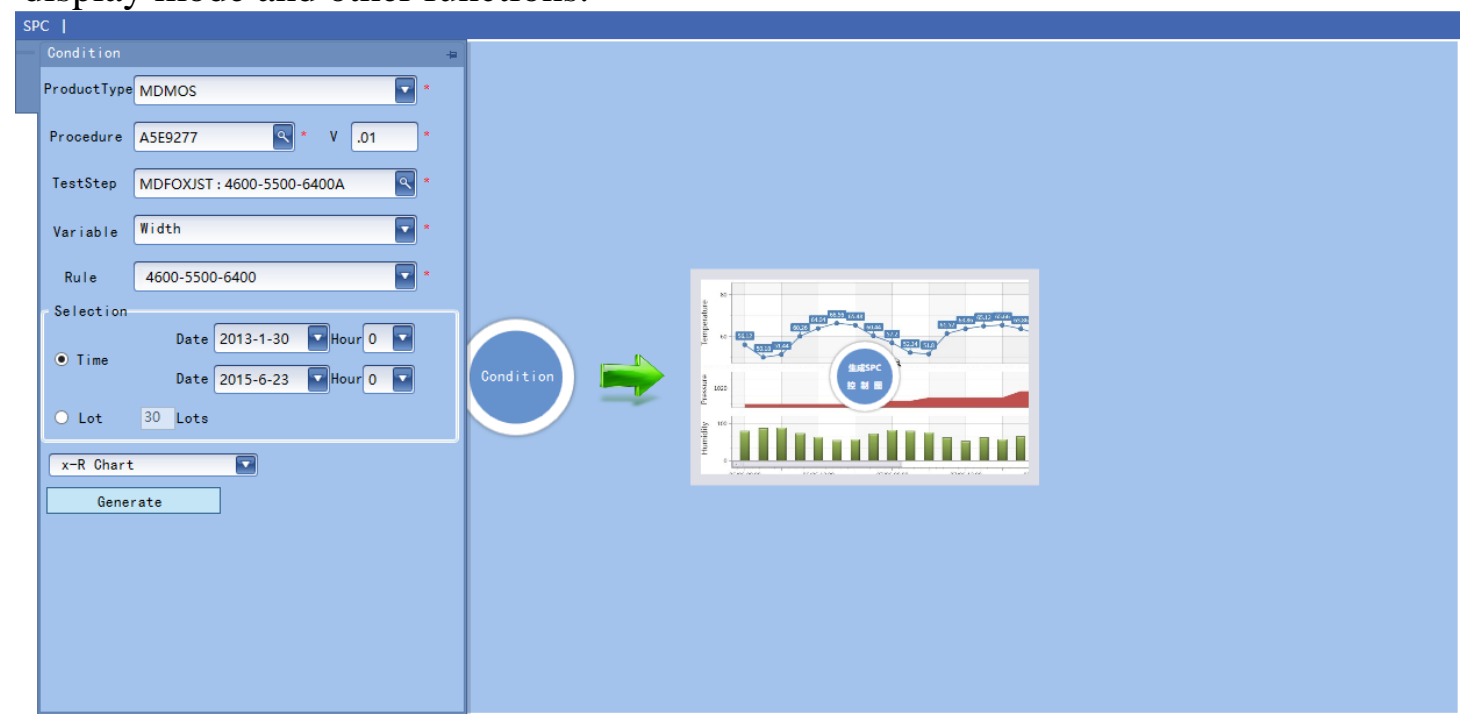

Fig. 5 SPC Variable

- Input production type, procedure, test step, variable, rules, query conditions, and select the required control chart, you can generate a qualified control chart.

This section contains SPC control chart according to "time query" and "according to the batch query, which according to" time query "for the period of time according to the batch data statistics," query "should be selected according to the number of batches of batches, batch number of the statistical data set in real time.

If offline SPC query, the selected conditions have not maintained the control line, pop-up temporary control line input window, where the control line data only as a temporary generation of useful, not saved to the database. Enter the temporary control line and determine the control chart of the filled control line.

- Right click on the control chart, the menu shown in the diagram. Includes control line display selection, change $\mathrm{Y}$ axis range, discrimination rule selection, Excel, grouping function. 


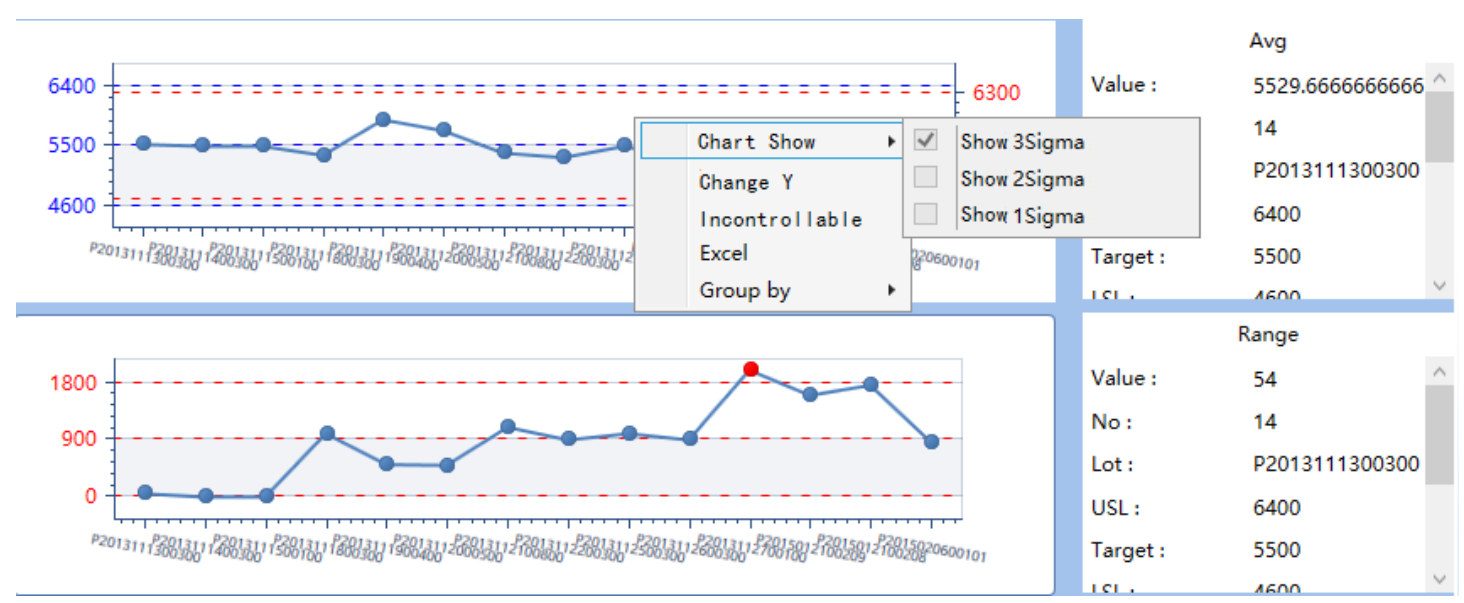

Fig. 6 SPC Chart Details

\section{Conclusion}

Based on the MES system, the realization of SPC control chart rendering and field management functions. Among them, the realization of a variety of control charts, can be applied to a variety of metering variables and counting variables. The disadvantage is that the dependence on the MES system is higher, the future research, hoping to be improved.

\section{Acknowledgement}

This work is supported by the Science and Technology Plan Project of Liaoning Province (2015106015) and the Key Laboratory of Network Control System, Chinese Academy of Sciences.

\section{References}

[1]Dai L W. Based on the Digital Design and Manufacture Technology of the Digital Workshop [J]. Journal of Anhui Vocational College of Metallurgy \& Technology, 2013.

[2]Sohal A S. Assessing manufacturing/quality culture and practices in Asian companies [J]. International Journal of Quality \& Reliability Management, 1998, 15(8/9):920-930.

[3]MORTON J. M. POSNER, CHARLES S. TAPIERO. Manufacturing quality density, control and maintenance [J]. International Journal of Production Research, 1987, 25(10):1511-1521.

[4]M. Colledani, T. Tolio. Integrated analysis of quality and production logistics performance in manufacturing lines [J]. International Journal of Production Research, 2011, 49(2):485-518.

[5]Goyal S K, Gunasekaran A, Martikainen T, et al. Integrating production and quality control policies: A survey[J]. European Journal of Operational Research, 1993, 69(1):1-13.

[6]Zorriassatine F, Tannock J D T. A review of neural networks for statistical process control [J]. Journal of Intelligent Manufacturing, 1998, 9(3):209-224.

[7]Lübeck M, Rodrigues T D. Research on manufacturing quality data management methods of quality statistical process control [J]. Computer Integrated Manufacturing Systems, 2005, 11(2):280-283.

[8]Mason B, Antony J. Statistical process control: an essential ingredient for improving service and manufacturing quality [J]. Measuring Business Excellence, 2001, 10(4):233-238. 